\title{
Preservation of post-transplant lung function with aerosol cyclosporin
}

\author{
T.E. Corcoran*, G.C. Smaldone*, J.H. Dauber*, D.A. Smith*, K.R. McCurry ${ }^{\Uparrow}$, G.J. Burckart", A. Zeevi ${ }^{+}$, \\ B.P. Griffith ${ }^{\S}$, A.T. lacono*
}

Preservation of post-transplant lung function with aerosol cyclosporin. T.E. Corcoran G.C. Smaldone, J.H. Dauber, D.A. Smith, K.R. McCurry, G.J. Burckart, A. Zeevi, B.P. Griffith, A.T. Iacono. (C) ERS Journals Ltd 2004.

ABSTRACT: Post-lung transplant use of aerosol cyclosporin (ACsA) is considered by examining the relationship between deposited aerosol dose and effect.

In a sub-study of placebo controlled trials of ACsA as a rejection prophylaxis, 15 drug subjects received aerosol dose quantification tests to gage their ability to effectively deposit the nebulised drug in their transplanted lung(s). A total of seven placebo subjects received mock deposition tests. The deposited doses and mock doses were compared to changes in the forced expiratory volume in one second, at six time points during the 2-yr trial period (ACsA was started within 6 weeks post-transplant).

Linear relationships were demonstrated between deposited dose and improvement in lung function in the drug subjects at all intervals. Mock dose data from placebo subjects did not demonstrate similar correlation. Based on these results, subjects were grouped by dose and compared. Subjects depositing $\geqslant 5 \mathrm{mg}$ of the drug in the periphery of their transplant(s) had improving pulmonary function on average. Low-dose and placebo subjects demonstrated declines, more $\mathrm{A2}-\mathrm{A4}$ rejection events in the latter portion of the trial, and more chronic rejection beyond the end of the trial.

A dose-to-effect relationship is demonstrated for aerosol cyclosporin in terms of pulmonary function and biopsy proven rejection.

Eur Respir J 2004; 23: 378-383.
*Division of Pulmonary, Allergy and Critical Care Medicine, "Division of Cardiothoracic Surgery, and ${ }^{+}$Division of Transplant Pathology, University of Pittsburgh, Pittsburgh, PA, \#Pulmonary/Critical Care Medicine, SUNY at Stony Brook, Stony Brook, NY, and ${ }^{\S}$ Division of Cardiac Surgery and Cardiopulmonary Transplantation, University of Maryland Medical Center, Baltimore, MD, USA.

Correspondence: T.E. Corcoran, UPMC MUH NW628, 3459 Fifth Ave., Pittsburgh, PA 15213, USA.

Fax: 14126477875

E-mail: corcorante@msx.upmc.edu

Keywords: Aerosol cyclosporin, aerosol deposition, lung transplantation

Received: May 282003

Accepted after revision: September 242003

This research was supported by grants from the National Heart, Lung and Blood Institute, and the American Lung Association. The cyclosporin powder was provided by Novartis Pharmaceuticals.
Rejection of the transplanted lung occurs at a rate exceeding that of most other solid organ allografts. Rejection of the allograft typically results in decreased pulmonary function and requires potent immunosuppressive treatments that predisposes the patient to opportunistic infections. Persistent acute rejection is the primary risk factor for bronchiolitis obliterans (OB), the pathological marker of chronic rejection [1]. Chronic rejection is the leading cause of late mortality in the lung-transplant population with a median survival after a diagnosis of $\sim 3$ yrs [2, 3].

The lung offers a unique opportunity for topical immunosuppression and the potential sparing of the significant sideeffects associated with systemic immunosuppressive agents. Aerosol (nebulised) cyclosporin (ACsA) has been studied for use as an adjuvant therapy for refractory acute rejection of the lung allograft. In two small open-label cohort studies, improvements in rejection grade and pulmonary function were noted after initiation of ACsA [4, 5]. ACsA was also used to stabilise pulmonary function in subjects suffering from chronic rejection [6].

A randomised, double-blind, placebo controlled trial of the prophylactic use ACsA was initiated to determine whether the drug is effective in preventing acute and chronic rejection. A deposition sub-study was later initiated to gage how well the subjects were depositing the study medication in their lungs. Deposition tests were performed on both drug and placebo subjects and both deposited and mock doses were calculated.
The authors hypothesised the following: 1) deposited dose would correlate to an improvement in lung function in the drug subjects but that mock dose would not correlate to change in the lung function in the placebo subjects; 2) subjects depositing the drug in sufficient quantity would demonstrate improved lung function and decreased levels of acute and chronic rejection when compared to placebo subjects.

\section{Material and methods}

The Institutional Review Board at the University of Pittsburgh approved both the performance of the prophylaxis trial of ACsA, and the deposition sub-study reported herein. Recruitment for the sub-study was performed by a blindednurse coordinator who contacted all subjects, actively participating in the prophylaxis trial, during the sub-study enrolment period. A total of 22 subjects were willing to enrol in the substudy and make themselves available at the testing centre for a one time aerosol deposition test. All subjects were outpatients and considered to be clinically stable at the time of testing. All subjects completed with informed consent.

All subjects within the prophylaxis trial began treatments within 6 weeks of transplantation. The drug group received ACsA (Novartis Pharmaceuticals, East Hanover, NJ, USA) dissolved in propylene glycol (concentration $62.5 \mathrm{mg} \cdot \mathrm{mL}^{-1}$ ). 
The placebo group received aerosol propylene glycol with $0.9 \%$ sodium chloride. An Aerotech II nebuliser was used (CIS-US Inc., Bedford, MA, USA) driven at $10 \mathrm{~L} \cdot \mathrm{min}^{-1}$ by tank air or a high flow compressor (DeVilbiss 8650D, Sunrise Medical HHG, Somerset, PA, USA). Treatments began with $1.6 \mathrm{~mL}(100 \mathrm{mg})$ of study medication, which was increased to $4.8 \mathrm{~mL}$ (300 mg) over a 10-12 day period. Subjects continued treatments, at their maximum, tolerated dose, for three times per week over 2 yrs. Subjects were offered an option to pretreat with nebulised lidocaine and albuterol if they found the inhaled study medication to be irritating. Otherwise all trial participants were treated identically based on the standard of care for lung transplant recipients at the University of Pittsburgh. Maintenance immunosuppression consisted of oral cyclosporin or tacrolimus, azathioprine or mycophenolate mofetil, and prednisone. Enhanced immune suppression for treatment of acute rejection and/or active OB consisted of pulse corticosteroids or cytolytics.

The radioisotope techniques used for deposited aerosol dose quantification have previously been described [5, 7]. To summarise, a known quantity of radioactive tag (Technetium $99 \mathrm{~m}$ bound to diethylenetriaminepentaacetic acid) was mixed into the study medication (drug or placebo) prior to nebulisation. The volume of study medication deposited within the lungs could then be determined after the treatment was inhaled. In subjects receiving aerosol cyclosporin, the mass of active drug deposited was then determined, based on the known concentration of the solution $\left(62.5 \mathrm{mg} \cdot \mathrm{mL}^{-1}\right)$. Bench testing, performed prior to these studies, demonstrated that the radioactivity associated with the tag, proportionally tracks the mass of active drug. In placebo subjects a mock dose was estimated, this was based on the volume of placebosolution deposited in the subject. This mock dose represents the dose of active drug that the subject would have received if the drug solution had been administered rather than placebo. Both the subjects and personnel performing the tests were blinded to the contents of the study medication.

Radioisotope deposition testing yields information on both total and regional deposited aerosol dose. The dose deposited can be divided into a central and a peripheral component, based on an area-convention which is applied to planar, gamma-camera images [5]. Left and right lung doses were averaged in double lung recipients so that transplant dose could be represented by a single quantity.

Pulmonary function data was extracted from a prospectively maintained clinical database at available points nearest to the day in question. Baseline pulmonary function was defined as each subject's best forced expiratory volume in one second (FEV1) value measured before postoperative day (POD) 100. This value was used instead of other common conventions based on the need to bench mark best lung function after postoperative recovery and before the subject had received the drug for an extended period. The percentage change in FEV1 from baseline was examined at POD 200, $300,400,500,600$ and 700. From the total of 15 drug subjects included in the data set, 11 were found to have reached day 700 at the time of analysis. No drug subjects died during the 2 -yr trial. However, two of the seven placebo subjects who received deposition tests died before day 400. A total of four, from the remaining five placebo subjects, had reached day 700 of the trial at the time of analysis.

Based on the demonstration of an apparent therapeutic dose of $5 \mathrm{mg}$ in the results taken from the above analysis, the subjects from the drug arm of the trial were divided into a high dose group $(\geqslant 5 \mathrm{mg}$ of deposited dose in the periphery of the transplanted lung, $\mathrm{n}=11)$, and a low dose group $(<5 \mathrm{mg}$, $\mathrm{n}=4$ ). The average change in FEV1 over time was compared between these groups. A comparison was also made with the average pulmonary function from all subjects in the placebo arm of the prophylaxis trial $(n=30)$.

Results from transbronchial biopsies were also collected in the high-dose, low-dose, and placebo groups. These biopsies were performed as part of normal post-transplant monitoring. The results were maintained prospectively in a clinical database. Typically surveillance biopsies were performed every 3-4 months during the first two post transplant years. Additional biopsies were performed as clinically indicated. Biopsy grading was done in accordance with standard conventions [8].

Only biopsy results were considered as a means of diagnosing acute or chronic rejection. Rejection events diagnosed through other clinical means were not included.

The number of grade A2 or greater events prior to POD 100 was determined for each subject and normalised by the number of biopsies performed during that period. This data was averaged for the high-dose, low-dose and placebo groups. This represents the level of rejection associated with the baseline value of FEV1. In a similar manner, the total number of rejection events (A1-A4) per biopsy, and the total number of grade A2 or greater events per biopsy was determined for each subject during the period of POD 100-700 and averaged.

The number of subjects receiving a histological diagnosis of chronic rejection $(\mathrm{C} 1, \mathrm{BO})$ prior to POD 700 was assessed in each group, as was the number of subjects receiving that diagnosis during or after the 2-yr trial, up until the point of analysis.

\section{Results}

The demographic and dose information from the 15 drug subjects who received deposition tests are shown in table 1 . Single lung recipients deposited between $2.2-9.2 \%$ of the drug added to the nebuliser in their transplanted lung, versus. $3.3-7.1 \%$ per transplanted lung in the doubles ( $\mathrm{p}=\mathrm{NS})$. Demographic and mock dose data for the seven placebo subjects, who received deposition tests, are presented in table 2 .

The relationship between peripheral/mock dose and the percentage improvement in lung function at POD 200, 300, 600 and 700 are shown in figure 1. The drug subjects depositing the highest doses in the periphery of their transplanted lung(s) demonstrated the largest improvement in lung function, especially at the latter time points of the trial.

The linear best fit data for change in FEV1 versus. peripheral transplant dose for the drug subjects, at all time points during the trial, are shown in table 3 . There was good correlation between dose and improvement in lung function in the drug subjects, at all time points considered (coefficient of determination: $\mathrm{r}^{2} ; 0.43-0.68 ; \mathrm{p}<0.01$ ). The slope of the best fit line represents the percentage gain in FEV1 per $\mathrm{mg}$ of deposited drug. This slope increased with POD increase, as illustrated in table 3 , indicating that the effects of the drug became more pronounced throughout the 2-yr trial. The $\mathrm{x}$-intercept of the best fit lines indicates the dose at which the subjects demonstrate no improvement or decline in their FEV1 value versus their baseline. In every case the best fit line intersected the $\mathrm{x}$-axis at $\sim 5 \mathrm{mg}$, indicating that doses $\geqslant 5 \mathrm{mg}$ in the periphery of the transplanted lung were likely to provide improvements in pulmonary function, whereas lesser doses did not.

Table 3 also includes the linear best fit data for correlations between whole transplant dose and improvement in lung function. The $r^{2}$ values associated with these correlations were not as strong, but a whole transplant protective dose of $\sim 12 \mathrm{mg}$ was apparent. This dose is lower than the whole lung therapeutic dose of $20 \mathrm{mg}$ reported by IACONO et al. [5] for the 


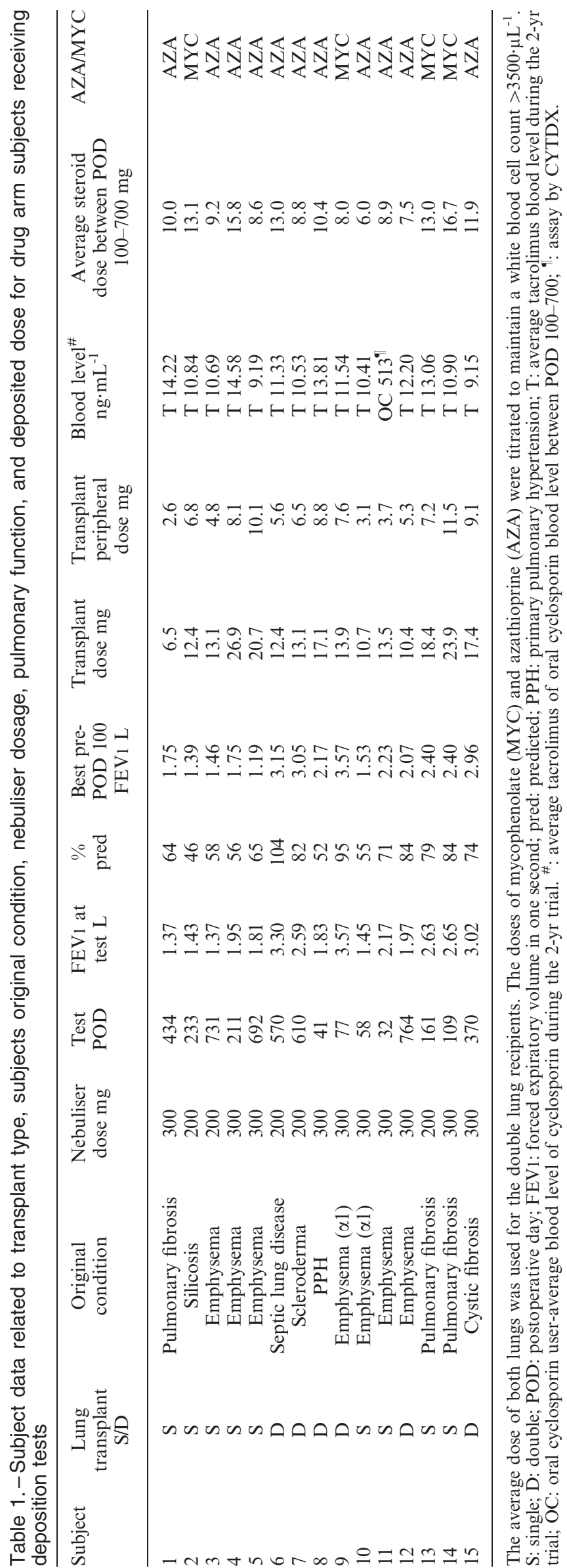

treatment of refractory rejection. Logically it might be expected that a higher dose would be required to reverse refractory-acute rejection versus preventing its initial onset. Better correlation between peripheral dose (versus whole lung dose) and effect would also be anticipated since the peripheral dose is less susceptible to mucociliary clearance and is more likely to have reached the whole volume of the lung.

Also included in figure 1 is the mock-dose data for the placebo subjects. Values of $\mathrm{r}^{2}$ were recalculated for a data set that included both drug-subject dose and placebo-subjectmock dose data at each time point. In all cases the addition of mock-dose data resulted in a significantly decreased $r^{2}$ value indicating that the placebo subject data failed to correlate with the drug-subject data. For days 200-700 these $\mathrm{r}^{2}$ values were $0.16,0.08,0.32,0.41,0.26$ and 0.33 compared to 0.43 , $0.56,0.61,0.62,0.67$ and 0.68 from the drug subjects alone. Mock dose by itself did not demonstrate substantial correlation with change in FEV1 $\left(\mathrm{r}^{2}=0.04,0.06,0.29,0.12\right.$, $0.17,0.11$ )

Figure 2 shows the average percentage change in pulmonary function for the group of subjects who deposited $\geqslant 5 \mathrm{mg}$ in the periphery of their transplanted lung(s) (the high dose group, $n=11)$. Data is also presented for recipients in the $<5 \mathrm{mg}$ low dose group $(\mathrm{n}=4)$, and recipients in the placebo arm of the study $(n=30)$. This placebo group includes data from all placebo subjects enroled in the prophylaxis trial whether they received a mock aerosol deposition test or not. Not all subjects had pulmonary function test PFT data available at each time point. No subjects in the drug-groups group died during the trial. Five placebo subjects died during the trial. The high-dose group demonstrated stable or slightly increasing pulmonary function throughout the trial, whereas the low-dose group and the placebo group demonstrated average declines. The rate of decline in the placebo group (the slope of the linear fit) was twice that of the low dose group (-0.02 versus -0.01 per cent change in FEV1 per POD). A twoway analysis of the variance (ANOVA) comparing percentage change in FEV1 of the high-dose drug group versus the placebo group at days 200-700 indicated significance with $\mathrm{p}=0.001$. An analysis considering only single lung transplant recipients from each of the groups for POD 200-600 produced similar results $(\mathrm{p}=0.002)$.

Biopsy results from the groups were considered to see if the changes in pulmonary function correlated to differences in acute or chronic rejection within the group (table 4). Data was included for the high-dose group, the low-dose group, and the placebo group. Statistical comparisons were made only between the high-dose group and the placebo group since the number of subjects in the low-dose group was minimal. The high-dose group and the placebo group had a similar average number of early (before POD 100) grade A2 or higher rejection events (normalised by the number of biopsies performed). Assessing rejection throughout the rest of the trial (POD 100-700), the groups had a similar number of total rejection events (A1-A4) per biopsy, but the average number of $\mathrm{A} 2$ or greater rejection events per biopsy was significantly lower in the high-dose ACsA group. The number and percentage of subjects receiving a histological diagnosis of chronic rejection during the trial was higher in the placebo subjects, though not statistically significant $(\mathrm{p}=0.09)$. When the period of observation was extended beyond the end of the trial, up until the day of analysis this result became significant $(p=0.04)$. This result is of course vulnerable to differences in follow up period. The average follow-up period to the day of analysis was longest for the low-dose drug group (1,772 days), followed by the high-dose group (1,261 days), and the placebo group (964 days). The average period of follow-up for placebo subjects alive at the day of analysis was 1,109 days. 
Table 2. - Subject data related to transplant type, subject, original condition, pulmonary function, and mock-dose data from placebo subjects who received deposition tests

\begin{tabular}{|c|c|c|c|c|c|c|c|c|c|c|c|}
\hline Subject & $\begin{array}{l}\text { Lung } \\
\text { transplant } \\
\text { S/D }\end{array}$ & $\begin{array}{l}\text { Original } \\
\text { condition }\end{array}$ & $\begin{array}{l}\text { Test } \\
\text { POD }\end{array}$ & $\begin{array}{l}\text { FEV1 } \\
\text { at test } \\
\mathrm{L}\end{array}$ & $\begin{array}{c}\% \\
\text { pred }\end{array}$ & $\begin{array}{l}\text { Best pre- } \\
\text { POD100 } \\
\text { FEV1 L }\end{array}$ & $\begin{array}{c}\text { Transplant } \\
\text { mock } \\
\text { dose mg }\end{array}$ & $\begin{array}{c}\text { Transplant } \\
\text { peripheral } \\
\text { mock dose } \\
\text { mg }\end{array}$ & $\begin{array}{c}\text { Blood level }^{\#} \\
\mathrm{ng} \cdot \mathrm{mL}^{-1}\end{array}$ & $\begin{array}{c}\text { Average } \\
\text { steroid dose } \\
\text { between POD } \\
100-700 \mathrm{mg}\end{array}$ & $\begin{array}{l}\text { AZA/ } \\
\text { MYC }\end{array}$ \\
\hline 16 & $\mathrm{~S}$ & Pulmonary fibrosis & 805 & 1.80 & 85 & 1.58 & 28.9 & 16.3 & T 13.27 & 12.5 & AZA \\
\hline 17 & $\mathrm{~S}$ & Emphysema & 745 & 1.38 & 64 & 1.05 & 26.8 & 12.5 & T 9.66 & 10.0 & AZA \\
\hline 18 & $\mathrm{~S}$ & Emphysema & 184 & 1.46 & 43 & 1.53 & 19.8 & 9.7 & Т 8.13 & 20.0 & AZA \\
\hline 19 & $\mathrm{~S}$ & Emphysema & 759 & 1.93 & 57 & 2.20 & 12.5 & 5.8 & Т 12.69 & 13.2 & AZA \\
\hline 20 & $\mathrm{~S}$ & Silicosis & 246 & 1.34 & 33 & 1.62 & 33.9 & 12.8 & Т 14.25 & 10.0 & MYC \\
\hline 21 & $\mathrm{~S}$ & Silicosis & 259 & 2.02 & 70 & 2.17 & 25.2 & 13.5 & Т 13.33 & 11.3 & AZA \\
\hline 22 & $\mathrm{D}$ & Cystic fibrosis & 584 & 3.34 & 81 & 3.09 & 19.1 & 8.7 & OC $180.8^{\bullet}$ & 15.0 & MYC \\
\hline
\end{tabular}

Mock dose is based on the deposited volume of placebo in the lungs and the concentration of the drug arm preparation. The average mock dose of both lungs was used for the double lung recipient. S: single; D: double; POD: postoperative day; FEV1: forced expiratory volume in one second; pred: predicted; AZA: azathioprine; MYC: mycophenolate; T: average tacrolimus blood level during the 2-yr trial; OC: oral cyclosporin user-average blood level of cyclosporin during the 2-yr trial. ${ }^{\#}$ : average tacrolimus of oral cyclosporin blood level between POD 100-700; ${ }^{\top}$ : assay by CYMONO.

\section{Discussion}

This study provides the first evidence of a deposited dose to effect relationship for ACsA when used as prophylaxis for lung allograft rejection. There was a positive relationship between deposited ACsA dose and improvement in lung function in 15 subjects during a 2 -yr post-operative course of the drug. The dose effecting a relationship, became significantly more pronounced over the course of the trial in a very predictable manner.

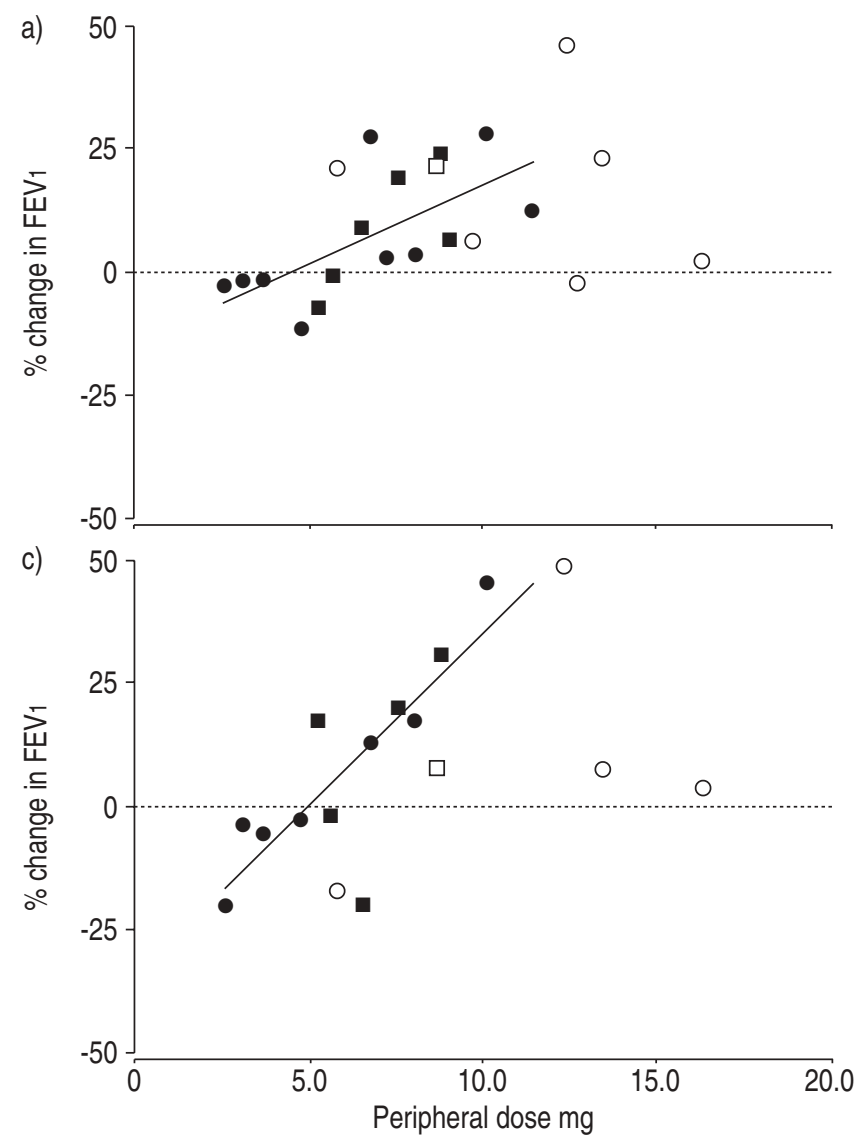

A very clear threshold dose of $5 \mathrm{mg}$ in the peripheral lung was established (table 3 and fig. 1). The subjects who deposited $\geqslant 5 \mathrm{mg}$ of the drug in the lung periphery demonstrated stability or improvement in pulmonary function over time, while those depositing $<5 \mathrm{mg}$ or receiving placebo demonstrated a decline. The rate of decline was steeper for the placebo group compared to the low-dose group. A survivor effect exists in the placebo group, due to the death of five subjects during the trial which is likely to underestimate the rate of decline. The effects demonstrated through pulmonary

b)

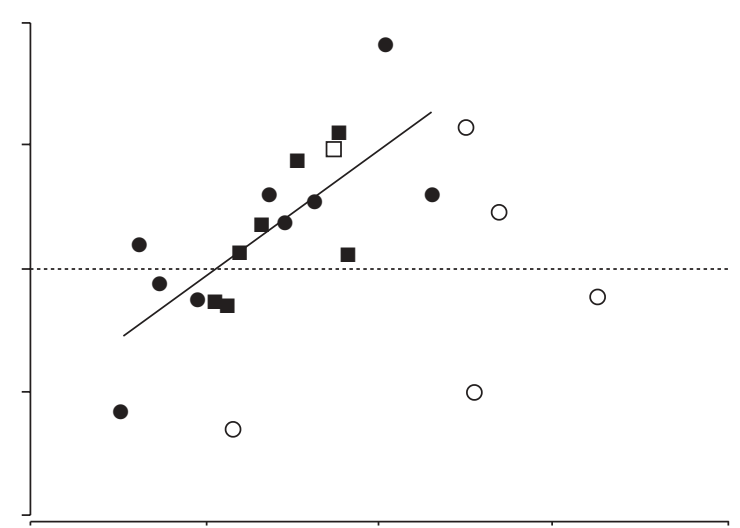

d)

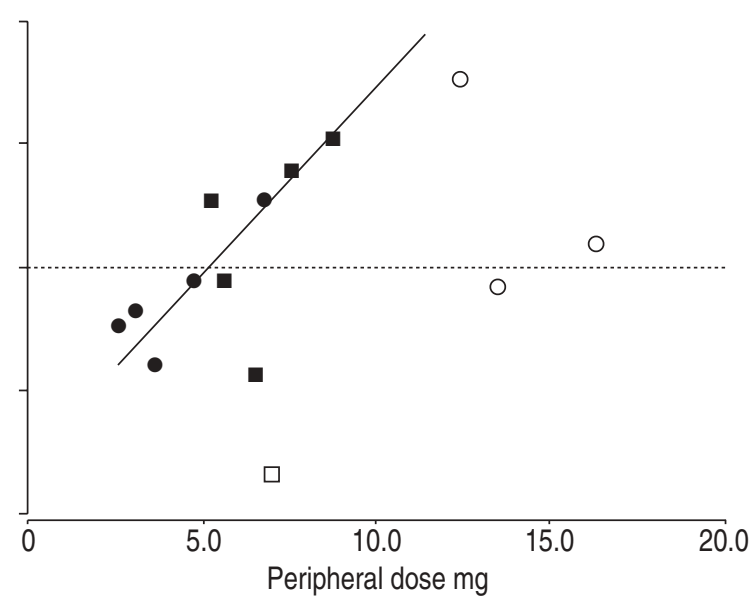

Fig. 1.-Relationship between dose deposited in the periphery of the transplanted lung(s) and improvement in forced expiratory volume in one second $\left(\mathrm{FEV}_{1}\right)$ at postoperative days a) 200 , b) 300, c) 600 and d) 700 . @: single lung with aerosolcyclosporin (ACsA); $\mathbf{0}$ : double lung with ACsA; $\bigcirc$ : single lung with placebo; $\square$ double lung with placebo. Linear relationships are depicted and the listed coefficient of determination values $\left(r^{2}\right)$ are for the drug-group only: a) $r^{2}=0.43$; $) r^{2}=0.56$; c) $r^{2}=0.67$; d) $r^{2}=0.68$. 
Table 3.-Data describing the relationship between transplant-peripheral and whole-transplant-deposited dose of aerosol cyclosporin and improvement in lung function at postoperative days 200-700

\begin{tabular}{|c|c|c|c|c|c|c|c|c|c|}
\hline \multirow[t]{2}{*}{ POD } & \multirow[t]{2}{*}{$\mathrm{n}$} & \multicolumn{4}{|c|}{ Peripheral lung dose } & \multicolumn{4}{|c|}{ Whole lung dose } \\
\hline & & $r^{2}$ & Slope & $\mathrm{x}$-intercept & p-value & $r^{2}$ & slope & $\mathrm{x}$-intercept & $\mathrm{p}$-value \\
\hline 200 & 15 & 0.43 & 3.16 & 4.53 & 0.008 & 0.12 & 0.80 & 6.70 & 0.206 \\
\hline 300 & 15 & 0.56 & 5.11 & 5.29 & 0.001 & 0.39 & 2.03 & 11.76 & 0.013 \\
\hline 400 & 14 & 0.61 & 5.92 & 5.06 & 0.001 & 0.54 & 2.55 & 11.68 & 0.003 \\
\hline 500 & 13 & 0.62 & 6.48 & 5.31 & 0.001 & 0.37 & 2.35 & 11.69 & 0.027 \\
\hline 600 & 12 & 0.67 & 7.03 & 5.02 & 0.001 & 0.38 & 2.34 & 11.03 & 0.033 \\
\hline 700 & 11 & 0.68 & 7.63 & 5.24 & 0.002 & 0.49 & 4.21 & 11.87 & 0.016 \\
\hline
\end{tabular}

The slope values represent the per cent increase in the forced expiratory volume in one second per mg of deposited drug. The $\mathrm{x}$-intercept values indicate the dose at which pulmonary function neither improves nor deteriorates. POD: postoperative day. Calculations of p-values are based on MOTULSKY [9].

function were associated with significant differences in rejection rates. Subjects depositing $\geqslant 5 \mathrm{mg}$ demonstrated lower levels of grade A2 or higher rejection during the later

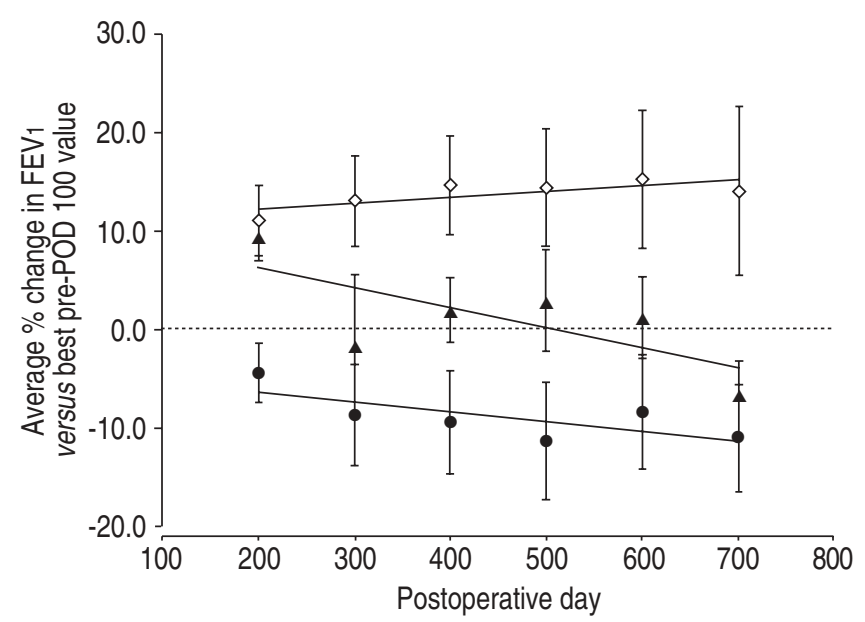

Fig. 2. - Average change in lung function for three subjects groups. $\diamond$ : subjects who deposited $\geqslant 5 \mathrm{mg}$ of aerosol cyclosporin (ACsA) in the periphery of their transplanted lung (high-dose group); $\mathbf{0}$ : those who deposited $<5 \mathrm{mg}$ of ACsA (low-dose group); $\boldsymbol{\Lambda}$ : placebo subjects. FEV1: forced expiratory volume in one second; POD: postoperative day. A two-way analysis of the variance comparing the high-dose group and the placebo group produced $\mathrm{p}=0.001$ when the effect of ACsA was considered. The error bars represent SEM. When only single lung recipients were compared, similar trends are noted with $\mathrm{p}=0.002$. portion of the trial (POD 100-700). There was significantly less chronic rejection in this group when results beyond the end of the trial were considered.

The possibility that good pulmonary function may simply result in higher deposited doses must be considered. If this were the case in the drug subjects, it would seem likely that subjects receiving aerosol placebo would demonstrate similar behaviour. However, the mock doses measured in these subjects failed to correlate to the relationships established in the drug subjects, and failed to demonstrate any independent relationship with change in pulmonary function, though total numbers were small. Furthermore, as seen in figure 3, there was no significant relationship between FEV1 deposited on test day and deposited dose in the drug subjects tested. There is no reason to believe that improving lung function in these subjects, over the course of a 2-yr trial, would result in a higher deposited dose when test-day lung function demonstrates no significant relationship to deposited dose. The possibility of a subtle feedback mechanism, where better drug deposition results in improved lung function, that in some way tends to sustain greater drug deposition, cannot be totally discounted.

The validity of a one-time dose assessment must be considered, as must the testing of subjects at various points during their 2-yr trial. Subject availability to the testing centre was limited, and this prevented the subjects being tested at a set postoperative day or days during the trial. Though ideally deposition would have been assessed at several time points for each subject, the authors believed that the four-fold variability in the inter-subject allograft cyclosporin dose likely outweighs the day-to-day differences in dose that each subject may experience.

Table 4.-Comparison of biopsy results from high-dose, placebo, and low-dose groups

\begin{tabular}{|c|c|c|c|c|}
\hline & \multicolumn{3}{|c|}{ Subject } & \multirow{2}{*}{$\begin{array}{l}\mathrm{p} \text {-value HD } \\
\text { versus placebo }\end{array}$} \\
\hline & HD & Placebo & LD & \\
\hline Subjects $n$ & 11 & $27^{\#}$ & 4 & \\
\hline Average rejection events $\geqslant 2$ per biopsy prior to POD 100 & $0.39 \pm 0.09$ & $0.36 \pm 0.06$ & $0.25 \pm 0.25$ & 0.79 \\
\hline Average rejection events per biopsy POD $100-700$ & $0.50 \pm 0.07$ & $0.53 \pm 0.04$ & $0.53 \pm 0.14$ & $0.72^{\bullet}$ \\
\hline Average rejection events $\geqslant 2$ per biopsy POD $100-700$ & $0.03 \pm 0.02$ & $0.14 \pm 0.03$ & $0.08 \pm 0.08$ & $0.01^{\bullet}$ \\
\hline $\begin{array}{l}\text { Subjects receiving histological diagnosis of chronic rejection } \\
\text { before POD } 700 \mathrm{n}(\%)\end{array}$ & $0(0)$ & $6(22)$ & $0(0)$ & $0.09^{+}$ \\
\hline $\begin{array}{l}\text { Subjects receiving histological diagnosis of chronic rejection } \\
\text { before or after POD } 700\end{array}$ & $0(0)$ & $8(30)$ & $1(25)$ & $0.04^{+}$ \\
\hline
\end{tabular}

Data are presented as mean \pm SEM unless otherwise stated. High-dose (HD) group: $\geqslant 5 \mathrm{mg}$ of aerosol cyclosporin (ACsA) deposition in periphery of transplanted lung(s); low-dose (LD) group: $<5 \mathrm{mg}$ of ACsA deposition in periphery of transplanted lung(s); placebo: all subjects in placebo arm of prophylaxis trial. \#: three patients that did not live to day 100 were not included. p-Values based on comparison of high-dose group versus placebos. : comparison using an unpaired t-test; ${ }^{+}$: comparison using Chi-squared analysis. 


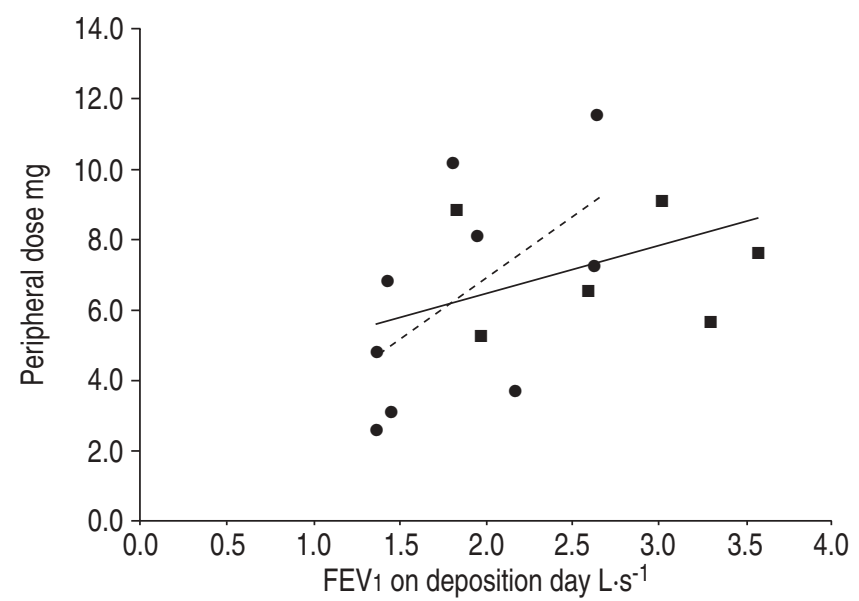

Fig. 3.- The relationship between peripheral transplant dose and the forced expiratory volume in one second (FEV1) in the aerosolcyclosporin (ACsA) administered subjects as measured on the day of deposition testing, no significance was found. 0 : single lung; double lung; - - -: single lung; —- all subjects. Single lung $\mathrm{r}^{2}=0.34$, $\mathrm{p}=\mathrm{NS}$; all subjects $\mathrm{r}^{2}=0.14, \mathrm{p}=\mathrm{NS}$.

Throughout several years of use ACsA has demonstrated relatively few side-effects. Past studies of ACsA for the treatment of acute rejection, reported no associated hepatotoxicity, nephrotoxicity, or post-transplant lymphoproliferative disease related to systemic absorption of the drug. The incidence of pneumonia was decreased with use of ACsA in these subjects [5]. Some subjects do experience shortness of breath, wheezing, or cough. In the high-dose group, six of the nine subjects who had adverse event data available reported at least one of these symptoms during the 2-yr trial, versus two of two in the low-dose group and 11 out of 30 of the placebo subjects. None of the drug subjects withdrew because of these symptoms.

Many factors contribute to the preservation of pulmonary function after lung transplantation. The addition of topical immunosuppression to currently accepted regimens of systemic immunosuppression appears here to tip the scales to a detectable degree, resulting in improved or sustained posttransplant lung function and corresponding decreases in several measures of acute and chronic rejection. These benefits, along with the lack of systemic effects associated with aerosol cyclosporin use, demonstrate the potential for topical immunosuppression in the field of lung transplantation.

\begin{abstract}
Acknowledgements. The authors would like to thank the following: D. Plaskon for his assistance in the performance of the deposition testing; $\mathrm{M}$. Brown, for his advice on the performance of these tests; R. Reissmann and L. Collins for their assistance with the deposition studies and C. Campbell and M. Williams for their assistance during the preparation of the manuscript.
\end{abstract}

\section{References}

1. Husain AN, Siddiqui MT, Holmes, et al. Analysis of risk factors for the development of bronchiolitis obliterans syndrome. Am J Respir Crit Care Med 1999; 159: 829-833.

2. Reichenspurner H, Girgis RE, Robbins RC, et al. Stanford experience with obliterative bronchiolitis after lung and heart-lung transplantation. Ann Thorac Surg 1996; 62: 14671473.

3. Valentine VG, Robbins RC, Berry GJ, et al. Actuarial survival of heart-lung and bilateral sequential lungtransplant recipients with obliterative bronchiolitis. $J$ Heart Lung Transplant 1996; 15: 371-383.

4. Keenan RJ, Iacono AT, Dauber JH, et al. Treatment of refractory acute rejection associated with aerosolized cyclosporine in lung transplant recipients. $J$ Thorac Cardiovasc Surg 1997; 113: 335-341.

5. Iacono AT, Smaldone GC, Keenan RJ, et al. Dose-related reversal of acute lung rejection by aerosolized cyclosporine. Am J Respir Crit Care Med 1997; 155: 1690-1698.

6. Iacono AT, Keenan RJ, Duncan SR, et al. Aerosolized cyclopsorine in lung recipients with refractory chronic rejection. Am J Respir Crit Care Med 1996; 153: 1451-1455.

7. Sangwan S, Agosti JM, Bauer LA, et al. Aerosolized protein delivery in asthma: gamma camera analysis of regional deposition and perfusion. J Aerosol Med 2001; 14: 185-195.

8. Yousem AA, Berry GJ, Cagle PT, et al. A revision of the 1990 working formulation of the classification of pulmonary allograft rejection: lung rejection study group. $J$ Heart Lung Transplant 1996; 15: 1-15.

9. Motulsky H. Intuitive Biostatistics. New York, Oxford University Press, 1995. 